\title{
Dielectric studies of binary mixtures of ethylene glycol mono phenyl ether with 1-butanol at different temperatures
}

\author{
H. A. Chaube* and V. A. Rana ${ }^{\dagger}$ \\ Department of Physics, School of Sciences \\ Gujarat University, Ahmedabad 380009 Gujarat, India \\ *chaubehemant099@gmail.com \\ †ranava2001@yahoo.com
}

Received 2 April 2017; Revised 4 June 2017; Accepted 4 July 2017; Published 1 August 2017

\begin{abstract}
Static permittivity $(\varepsilon)$, refractive index $(n)$ and density $(\rho)$ of binary mixtures of ethylene glycol mono phenyl ether (EGMPE) with 1-butanol $(1-\mathrm{BuOH})$ over the entire range of mole fraction and at temperatures $(T=303.15,313.15$ and $323.15 \mathrm{~K})$ have been measured. From the experimental data, parameters such as excess static permittivity $\left(\varepsilon^{E}\right)$, excess permittivity at optical frequency $\left(\varepsilon_{\infty}^{E}\right)$, effective Kirkwood correlation factor $\left(g^{\text {eff }}\right)$, corrective Kirkwood correlation factor $\left(g^{f}\right)$ and Bruggeman factor $\left(f^{b}\right)$ have been calculated to obtain qualitative and quantitative information about the complex formation through H-bond in binary system. In order to predict the static permittivity of polar-polar binary mixtures six mixing rules were applied and for refractive index five mixing rule were applied. Experimental results of permittivity $(\varepsilon)$ and refractive index $(n)$ are compared with those obtained from theoretical calculations. Excess parameters were fitted to the Redlich-Kister type polynomial equation.
\end{abstract}

Keywords: Static permittivity; refractive index; Kirkwood correlation factor; permittivity models; refractive index models; RedlichKister equation.

\section{Introduction}

The static permittivity is a macroscopic quantity which plays an important role in understanding the solution properties for associative polar molecules as well as for polymers. ${ }^{1-3}$ The static permittivity has proven to be a useful property in characterizing molecular interaction and molecular ordering in solutions, as the value of dielectric permittivity is strongly related both to the chemical structure of a molecule and to intermolecular interactions. ${ }^{4-7}$ By using dielectric data of binary liquid mixtures over a range of mole fractions and temperatures, it is possible to obtain valuable information regarding the nature and strength of interactions in liquid mixtures.

Further, precise experimental dielectric data of the polar liquids in mixed state over a range of concentration and temperature can help to establish correlation equations, which can help to predict the dielectric permittivity value at a given concentration or at a given temperature. ${ }^{4}$

Aim of the present investigation is to gain information about the modifications of molecular structures and dipolar orientation due to mixing of ethylene glycol mono phenyl ether (EGMPE) and 1-butanol (1-BuOH) over a complete range of concentrations, through dielectric measurements at different temperatures $(T=303.15,313.15$ and $323.15 \mathrm{~K})$. Recently, we studied dielectric, physico-chemical and ultrasonic properties of mixtures of ethylene glycol mono phenyl ether with 1-hexanol through entire concentration range at different temperatures. ${ }^{8}$ So, this study will provide additional information about the effect of change in carbon chain length of the alcohol on the dielectric properties of mixture of EGMPE and alcohol. Furthermore, literature survey suggests that the dielectric study of the binary mixtures of ethylene glycol mono phenyl ether (EGMPE) and 1-butanol (1-BuOH) in pure liquid state have not been studied so far.

\section{Materials, Methods and Evaluation of Different Parameters}

EGMPE (extra pure), 1-BuOH (AR grade) were obtained from HPLC Pvt. Ltd (India) and were used without further purification. Binary mixtures of EGMPE with 1-BuOH were prepared at nine concentrations by volume. The concentration was then converted into the mole fraction of component 1 , using following formula, ${ }^{9}$

$$
x_{1}=\frac{\rho_{1} \times\left(\frac{v_{1}}{M_{1}}\right)}{\rho_{1} \times\left(\frac{v_{1}}{M_{1}}\right)+\rho_{2} \times\left(\frac{v_{2}}{M_{2}}\right)},
$$

where, $M$ is molecular weight, $v$ is volume and $\rho$ is density. 1 represents $1-\mathrm{BuOH}$ and 2 represent EGMPE. The mole fraction is accurate to $0.1 \%$.

\footnotetext{
${ }^{\dagger}$ Corresponding author.
}

This is an Open Access article published by World Scientific Publishing Company. It is distributed under the terms of the Creative Commons Attribution 4.0 (CC-BY) License. Further distribution of this work is permitted, provided the original work is properly cited. 
Static permittivity, refractive index and density of EGMPE, 1-BuOH and their mixtures were determined by the method explained in our earlier work. ${ }^{9}$ From the experimental data, excess parameters such as excess static permittivity $\left(\varepsilon^{E}\right)$, excess permittivity at optical frequency $\left(\varepsilon_{\infty}^{E}\right)$ as well as Kirkwood correlation factor $(g)$, effective Kirkwood correlation factor ( $\left.g^{\text {eff }}\right)$, corrective Kirkwood correlation factor $\left(g^{f}\right)$, Bruggeman factor $\left(f^{B}\right)$ were evaluated using equations given in our previous paper. ${ }^{9,10}$

The variations of excess properties with composition are expressed by Redlich-Kister polynomial equation ${ }^{11}$

$$
Q=x_{1} x_{2} \sum_{r=0}^{n} a_{r}\left(x_{2}-x_{1}\right)^{r},
$$

where $Q$ refers all excess parameters. The adjustable parameters $a_{r}$, were determined by least square fitting methods.

\subsection{Mixing rules}

\subsubsection{Mixing rules for refractive index}

For binary mixtures of EGMPE $+1-\mathrm{BuOH}$ various mixing rules for predicting the refractive index $(n)$ given below were used,

Eyring-John (EJ) ${ }^{12}$

$$
n_{m}=n_{1} \phi_{1}^{2}+n_{21} \phi_{2}^{2}+2\left(n_{1} n_{2}\right)^{\frac{1}{2}} \phi_{1} \phi_{2},
$$

Gladestone-Dale (GD) $)^{13}$

$$
n_{m}-1=\left(n_{1}-1\right) \phi_{1}+\left(n_{2}-1\right) \phi_{2},
$$

Newton $(\mathrm{Nw})^{14}$

$$
n_{m}^{2}-1=\left(n_{1}^{2}-1\right) \phi_{1}+\left(n_{2}^{2}-1\right) \phi_{2},
$$

Argo-Biot $(\mathrm{AB})^{15}$

$$
n_{m}=n_{1} \phi_{1}+n_{2} \phi_{2},
$$

Heller $(\mathrm{H})^{16}$

$$
\frac{\left(n_{m}-n_{1}\right)}{n_{1}}=\frac{3}{2}\left(\frac{\left(\frac{n_{2}}{n_{1}}\right)^{2}-1}{\left(\frac{n_{2}}{n_{1}}\right)^{2}+2}\right) \phi_{2},
$$

where in Eqs. (3) to (7), $n$ is refractive index and $\phi$ is volume fraction. Suffix $m, 1$ and 2 represents mixture, $1-\mathrm{BuOH}$ and EGMPE, respectively.

\subsubsection{Mixing rules for permittivity}

Permittivity values of binary mixtures of EGMPE $+1-\mathrm{BuOH}$ were compared to those estimated by five mixing rules, which were proposed by:

Looyenga $^{17}$

$$
\varepsilon=\left[\varepsilon_{1}^{\frac{1}{3}}+\phi_{2}\left(\varepsilon_{2}^{\frac{1}{3}}-\varepsilon_{1}^{\frac{1}{3}}\right)\right]^{3} .
$$

Iglesias-Peon $^{18}$

$$
\varepsilon_{e}=\varepsilon_{\mathrm{id}}\left[1-\frac{2}{3}\left(\frac{1+\phi_{1}\left(\varepsilon_{1} / \varepsilon_{2}-1\right)}{\left(\varepsilon_{1} / \varepsilon_{2}\right)^{\phi_{1}}}\right)\right] .
$$

Kraszewski et al. ${ }^{19}$

$$
\varepsilon^{\frac{1}{2}}=\phi_{1} \varepsilon_{1}^{\frac{1}{2}}+\phi_{2} \varepsilon_{2}^{\frac{1}{2}} .
$$

Böttcher-Bordewijk ${ }^{20}$

$$
\frac{3 \varepsilon_{1}}{2 \varepsilon+\varepsilon_{1}} \phi_{1}+\frac{3 \varepsilon_{2}}{2 \varepsilon+\varepsilon_{2}} \phi_{2}=1 .
$$

Bruggeman asymmetric ${ }^{21}$

$$
\frac{\varepsilon_{2}-\varepsilon}{\left(\varepsilon / \varepsilon_{1}\right)^{1 / 3}}=\left(1-\phi_{2}\right)\left(\varepsilon_{2}-\varepsilon_{1}\right) \text {. }
$$

Lichtenecker-Rother ${ }^{22}$

$$
\varepsilon=\varepsilon_{1}^{\phi_{1}} \varepsilon_{2}^{\phi_{2}},
$$

where in Eqs. (8) to (13), $\varepsilon$ is static permittivity and $\phi$ is volume fraction. Suffix 1 and 2 represents $1-\mathrm{BuOH}$ and EGMPE, respectively.

The root mean square deviation (RMSD) values for the above mixing rules were determined by the following equation, ${ }^{23}$

$$
\mathrm{RMSD}=\left[\frac{1}{p} \sum\left(A_{\mathrm{exp}}-A_{\mathrm{cal}}\right)^{2}\right]^{\frac{1}{2}},
$$

where $A_{\exp }$ represents experimental permittivity and refractive index, $A_{\text {cal }}$ represents calculated permittivity and refractive index and $p$ represents total number of concentrations.

\section{Results and Discussion}

Comparison of static permittivity $(\varepsilon)$, permittivity at optical frequency $\left(\varepsilon_{\infty}\right)$, density $(\rho)$ and Kirkwood correlation factor $(\mathrm{g})$ of $1-\mathrm{BuOH}$ with literature data at $303.15 \mathrm{~K}$ is given in Table 1. Experimentally measured static permittivity $(\varepsilon)$, permittivity at optical frequency $\left(\varepsilon_{\infty}\right)$, density $(\rho)$, effective

Table 1. Comparison of static permittivity $(\varepsilon)$, permittivity at optical frequency $\left(\varepsilon_{\infty}\right)$, density $(\rho)$ and Kirkwood correlation factor $(g)$ of 1-BuOH with literature data at $T=303.15 \mathrm{~K}$.

\begin{tabular}{lcc}
\hline Property & 1-BuOH (Exp.) & 1-BuOH (Lit.) \\
\hline Static permittivity $(\varepsilon)$ & 16.69 & $16.91^{1}$ \\
& & $17.61^{24}$ \\
Permittivity at optical freq. $\left(\varepsilon_{\infty}\right)$ & 1.9485 & $1.9490^{25}$ \\
& & $1.9527^{1}$ \\
Density $(\rho) / \mathrm{Kg} \mathrm{m}^{-3}$ & 802.2 & $802.4^{26}$ \\
& & $802.2^{27}$ \\
Kirkwood correlation factor $(\mathrm{g})$ & 1.68 & $802.5^{28}$ \\
\hline
\end{tabular}

Note: $298.15 \mathrm{~K}$. 
Table 2. Experimentally measured static permittivity $(\varepsilon)$, permittivity at optical frequency $\left(\varepsilon_{\infty}\right)$, density $(\rho)$, effective Kirkwood correlation factor $\left(g^{\text {eff }}\right)$, corrective Kirkwood correlation factor $g^{f}$ and Bruggeman factor $\left(f^{B}\right)$ of binary mixtures of EGMPE +1-BuOH at Temp. $(T=303.15,313.15,323.15 \mathrm{~K})$.

\begin{tabular}{lclllll}
\hline$x_{1}$ & $\varepsilon$ & \multicolumn{1}{c}{$\varepsilon_{\infty}$} & $g^{\text {eff }}$ & $g^{f}$ & density $(\rho) / \mathrm{Kgm}^{-3}$ & $f_{B}$ \\
\hline & & & \multicolumn{5}{c}{ Temp. $=303.15 \mathrm{~K}$} & & \\
0.000 & 10.90 & 2.2593 & 2.654 & 1.000 & 1.093 & 1.000 \\
0.133 & 11.42 & 2.2351 & 2.658 & 0.960 & 1.064 & 0.896 \\
0.256 & 11.96 & 2.2121 & 2.673 & 0.935 & 1.029 & 0.792 \\
0.371 & 12.50 & 2.1867 & 2.702 & 0.921 & 0.994 & 0.691 \\
0.479 & 13.05 & 2.1589 & 2.744 & 0.915 & 0.961 & 0.592 \\
0.579 & 13.62 & 2.1289 & 2.797 & 0.916 & 0.930 & 0.492 \\
0.674 & 14.20 & 2.0954 & 2.863 & 0.923 & 0.900 & 0.394 \\
0.763 & 14.79 & 2.0603 & 2.940 & 0.936 & 0.873 & 0.296 \\
0.846 & 15.40 & 2.0217 & 3.033 & 0.954 & 0.847 & 0.199 \\
0.925 & 16.04 & 1.9854 & 3.133 & 0.975 & 0.825 & 0.099 \\
1.000 & 16.69 & 1.9485 & 3.241 & 1.000 & 0.802 & 0.000 \\
& & & Temp. $=313.15 \mathrm{~K}$ & & \\
0.000 & 10.57 & 2.25 & 2.447 & 1.000 & 1.083 & 1.000 \\
0.133 & 11.07 & 2.2199 & 2.446 & 0.960 & 1.050 & 0.894 \\
0.256 & 11.58 & 2.1936 & 2.456 & 0.935 & 1.016 & 0.791 \\
0.371 & 12.09 & 2.1697 & 2.476 & 0.919 & 0.984 & 0.689 \\
0.479 & 12.62 & 2.143 & 2.513 & 0.913 & 0.952 & 0.588 \\
0.579 & 13.16 & 2.1157 & 2.559 & 0.914 & 0.921 & 0.488 \\
0.674 & 13.70 & 2.0853 & 2.618 & 0.921 & 0.893 & 0.390 \\
0.763 & 14.26 & 2.0502 & 2.692 & 0.935 & 0.867 & 0.292 \\
0.846 & 14.84 & 2.0148 & 2.777 & 0.953 & 0.843 & 0.193 \\
0.925 & 15.42 & 1.9814 & 2.864 & 0.974 & 0.818 & 0.096 \\
1.000 & 16.02 & 1.9427 & 2.967 & 1.000 & 0.795 & 0.000 \\
& & & Temp. $=323.15 \mathrm{~K}$ & & \\
0.000 & 10.18 & 2.2404 & 2.295 & 1.000 & 1.073 & 1.000 \\
0.133 & 10.58 & 2.21 & 2.265 & 0.966 & 1.041 & 0.898 \\
0.256 & 10.98 & 2.1828 & 2.250 & 0.943 & 1.008 & 0.796 \\
0.371 & 11.39 & 2.1554 & 2.250 & 0.930 & 0.975 & 0.696 \\
0.479 & 11.81 & 2.1307 & 2.261 & 0.923 & 0.944 & 0.595 \\
0.579 & 12.24 & 2.1029 & 2.284 & 0.924 & 0.914 & 0.495 \\
0.674 & 12.68 & 2.0728 & 2.320 & 0.930 & 0.887 & 0.395 \\
0.763 & 13.13 & 2.0419 & 2.365 & 0.942 & 0.861 & 0.295 \\
0.846 & 13.59 & 2.008 & 2.422 & 0.958 & 0.836 & 0.196 \\
0.925 & 14.06 & 1.9726 & 2.486 & 0.978 & 0.811 & 0.097 \\
1.000 & 14.53 & 1.9379 & 2.554 & 1.000 & 0.786 & 0.000 \\
\hline & & & & &
\end{tabular}

Kirkwood correlation factor $\left(g^{\text {eff }}\right)$, corrective Kirkwood correlation factor $g^{f}$ and Bruggeman factor $f^{B}$ of binary mixtures of EGMPE +1 -BuOH at temperature $(T=303.15$, $313.15,323.15 \mathrm{~K})$ are presented in Table 2.

Static permittivity of EGMPE $(10.90$ at $303.15 \mathrm{~K})$ is smaller than the static permittivity of $1-\mathrm{BuOH}$ (16.69 at $303.15 \mathrm{~K}$ ), this difference in static permittivity values of these two molecules is in agreement with the difference in their molecular size (molar volume of EGMPE $\approx 1.25 \times 10^{-4} \mathrm{~m}^{3}$ $\mathrm{mol}^{-1}$ and of $\left.1-\mathrm{BuOH} \approx 0.92 \times 10^{-4} \mathrm{~m}^{3} \mathrm{~mol}^{-1}\right)$.

Plots of static permittivity $(\varepsilon)$ against $x_{1}$ (Fig. 1), show two distinct regions in the range $0<x_{1}<0.6$ and $0.6<x_{1}<1$ at all three temperatures. The boundary value of $x_{1}$ of these regions is highlighted by vertical dotted line in Fig. 1.

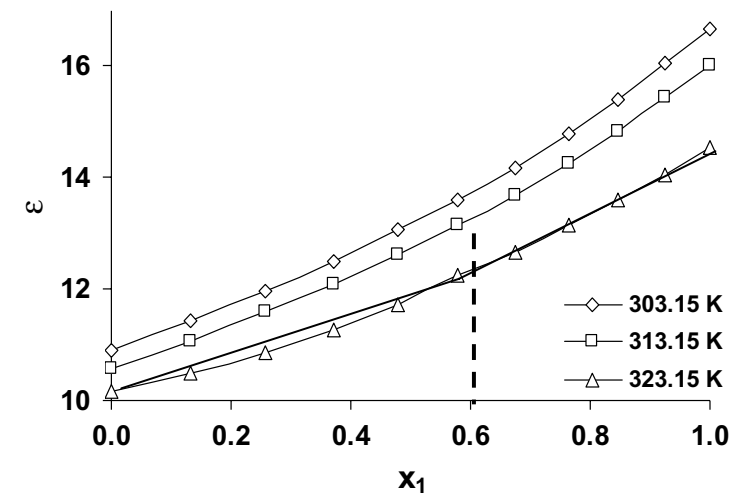

Fig. 1. Plot of static permittivity against $x_{1}$. (-) show smooth line joining data points. 
The $\varepsilon$ values vary nonlinearly with $x_{1}$ in the $0<x_{1}<0.6$ (EGMPE rich region) range but in the $0.6<x_{1}<1(1-\mathrm{BuOH}$ rich region) range linear behavior is observed. The linear region indicates the uniform cooperative behavior of the EGMPE-1-BuOH structures and also suggests that the state of EGMPE and 1-BuOH molecules does not change despite the large mixing range. Nonlinear mixing range $0<x_{1}<0.6$ suggests that the molecular structures of EGMPE and 1$\mathrm{BuOH}$ are different from their pure liquid state. In case of mixture of EGMPE and 1-hexanol two similar distinct regions were separated at 0.5 mole fraction of 1-hexanol. ${ }^{8}$

In the binary mixtures of polar liquids varieties of molecular interactions such as hydrogen bonding, molecular association, dipole-dipole and dipole- induced dipole interactions between the constituents are possible. As a result of these interactions, deviations occur from ideal behavior of static permittivity with concentration variation of the mixture constituents. ${ }^{29}$ The character changes of the static permittivity are very clear as the function of concentration of mixtures in case of excess values of $\varepsilon$. It can be seen from Fig. 2, that the $\varepsilon^{E}$ values for EGMPE +1 -BuOH mixtures are negative over the entire concentration range. The negative $\varepsilon^{E}$ values suggest that the polar associations are formed with lower dipole moments due to orientation of some of the neighboring dipoles in the opposite direction.

The different strength of H-bond interactions and net dipolar alignments is responsible for the divergent magnitude of $\varepsilon^{E}$ values at different temperatures. ${ }^{30}$ The magnitude of $\varepsilon^{E}$ can be ordered with temperature variation as $\varepsilon^{E} 323.15 K>\varepsilon^{E} 313.15 K>\varepsilon^{E} 303.15 K$. This clearly suggest that hydrogen bonded networks are more effectively dissociated.

Excess permittivity at optical frequency $\left(\varepsilon_{\infty}^{E}\right)$ were evaluated to examine the effect of heterogeneous H-bond on electronic polarization and plotted against mole fraction of $1-\mathrm{BuOH}$ as shown in Fig. 3. From the figure, it can be seen that excess permittivity at optical frequency $\left(\varepsilon_{\infty}^{E}\right)$ is positive over the entire range of concentration of 1-BuOH. Similar

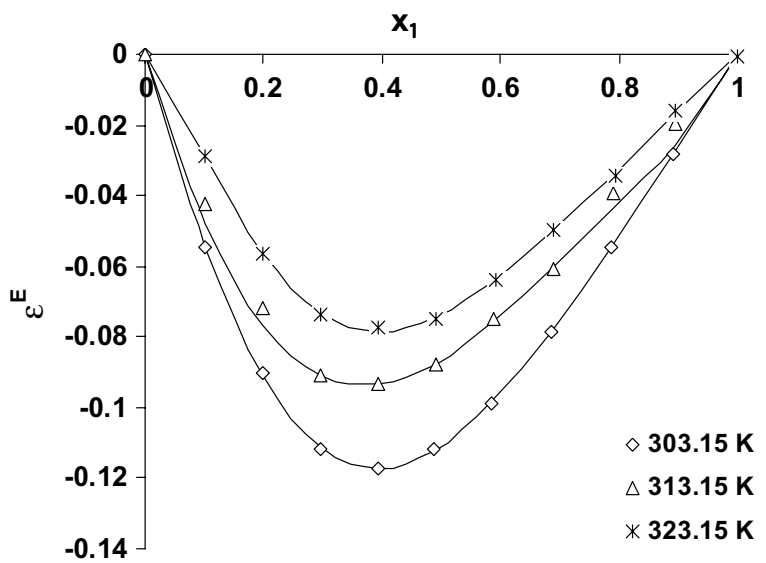

Fig. 2. Plot of excess static permittivity $\left(\varepsilon^{E}\right)$ against $x_{1}$. (-) show the fitted line of Redlich-Kister equation.

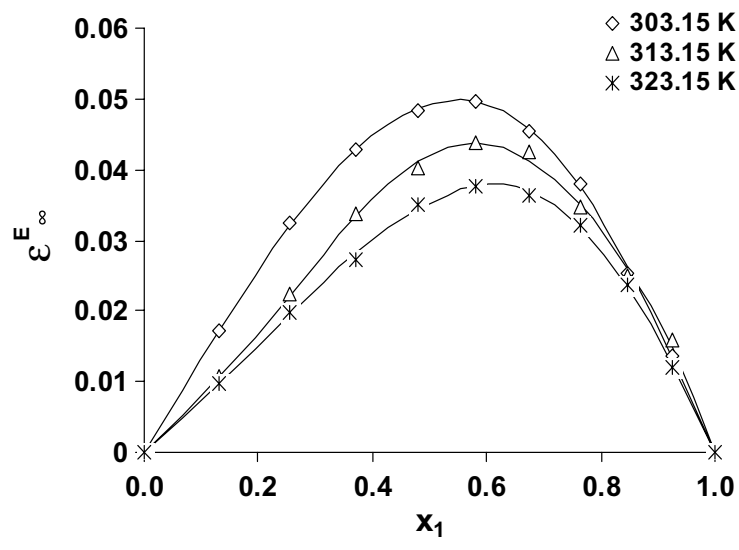

Fig. 3. Plot of excess permittivity at optical frequency $\left(\varepsilon_{\infty}^{E}\right)$ against $x_{1}$. (-) show the fitted line of Redlich-Kister equation.

trend is observed at all the three temperatures. This clearly indicates that the electronic polarization is influenced by the H-bonded complexation between the constituents of the binary mixture. ${ }^{31}$

Binary mixtures of polar liquids that have significant difference in molecular size and structures are the subject of interest to many researches. In such binary mixtures, varieties of molecular interaction among molecular species are taking place, not only that but the dominances of a particular type of interaction changes with concentration. ${ }^{4}$ Because of these, measured values of refractive index and static permittivity of binary mixture of polar liquids deviate significantly from the values evaluated using molar average values of the pure components. Hence, it is essential to verify various mixing rules for predicting refractive index and static permittivity value of a given binary liquid system and identify a particular role which provides the best agreement with the experimental results.

In the present investigation, we have tested five mixing rules for refractive index proposed by LL, EJ, W, GD, $\mathrm{Nw}, \mathrm{AB}$, and $\mathrm{H}^{12-16}$ and six mixing rules for static permittivity proposed by Looyenga, Kraszewski, Lglesias-Peon, Lichtenecker-Rotter, Bottcher and Bruggeman. ${ }^{18-22}$ Our experimental values of refractive index are compared with the predicted values using these rules at $303.15 \mathrm{~K}$ temperature in Table 3. The comparison between experimental and theoretical values of static permittivity for binary mixtures of EGMPE $+1-\mathrm{BuOH}$ using the predictive models at ( $T=303.15 \mathrm{~K}$ ) is shown in (Fig. 4).

The predictions of the mixing rules mentioned above for static permittivity are substantially equivalent; except the equation of Bruggeman that shows large deviation than the other ones. Equation of Rother, Bottcher, Looyenga and Iglesias are the models of low fluctuations and the permittivity of the mixture is near to the experimental values (Fig. 4), Kraszewski model provides best prediction for static permittivity. RMSD values of different mixing rules at $303.15 \mathrm{~K}$ are given in Table 4. 


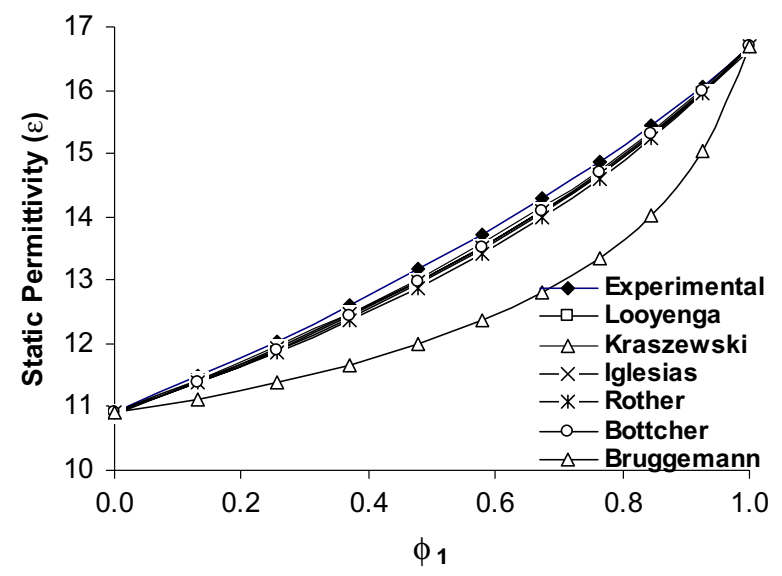

Fig. 4. Plot of experimental values of static permittivity $(\varepsilon)$ with predicted values by Looyenga, Kraszewski, Lglesias-Peon, Lichtenecker-Rotter, Bottcher and Bruggeman rules at $303.15 \mathrm{~K}$ against $\phi_{1}$.

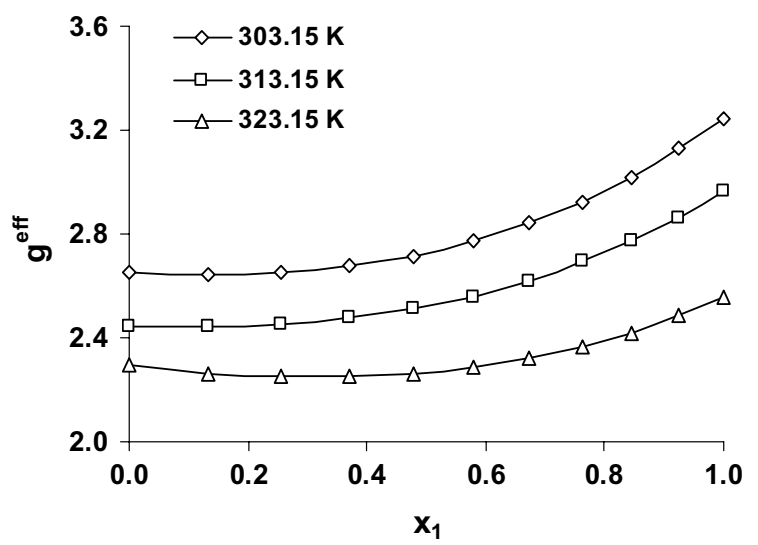

Fig. 5. Plot of effective Kirkwood co-relation factor $\left(g^{\text {eff }}\right)$ against $x_{1}$.

Determined values of Kirkwood co-relation factor is plotted against mole fraction of $1-\mathrm{BuOH}$ and is shown in Fig. 5. Information regarding orientation of electric dipoles in polar liquids can be obtained from the Kirkwood correlation factor $\left(g^{\text {eff }}\right)$. For both pure EGMPE and 1-BuOH, $g^{\text {eff }}$ values are greater than unity, which indicates that H-bonded molecular multimers of pure EGMPE and 1-BuOH favor parallel orientation.

From Fig. 5 it can be observed that $g^{\text {eff }}$ values of binary mixtures are found to vary from its value for pure $1-\mathrm{BuOH}$ to that of EGMPE. It can also be seen from figure that, the $g$ eff values vary with change in temperature, indicating that temperature change produces appreciable structural change.

The corrective Kirkwood correlation factor $g^{f}$ gives information about heterogeneous interaction between the components in the mixture (Fig. 6).

For an ideal noninteracting mixture, $g^{f}$ must be unity and a deviation of $g^{f}$ from unity indicates the existence of heterogeneous interaction between the components in the mixture. The value of $g^{f}$ depends on the concentration and

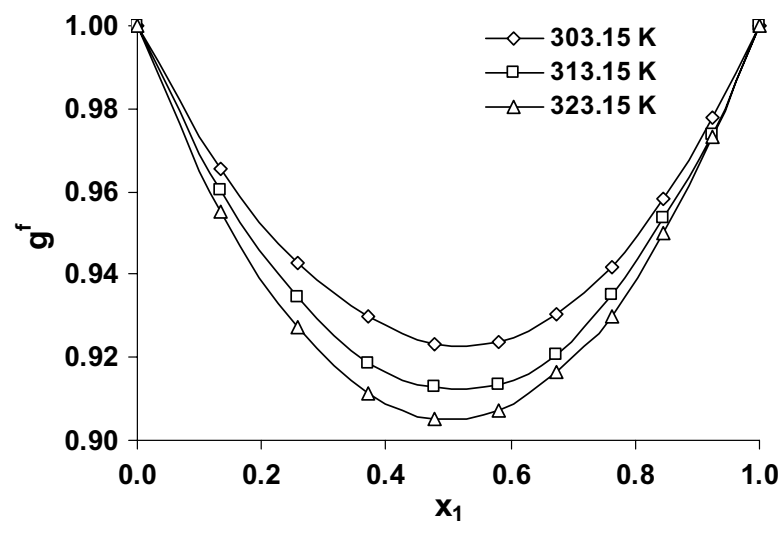

Fig. 6. Plot of corrective effective $\left(g^{f}\right)$ Kirkwood corelation factor against $x_{1}$.

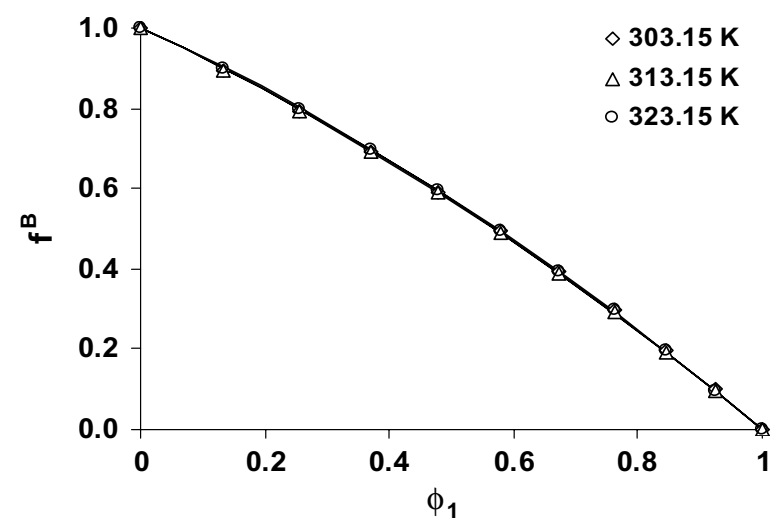

Fig. 7. Plot of Bruggeman factor $\left(f^{B}\right)$ against $\varphi_{1 .}(-)$ show the fitted line of Bruggeman equation.

the temperature. Krishnan et al. ${ }^{32}$ noticed that the value of $g^{f}$ will remain close to unity if there is no interaction between the component molecules. If the value of $g^{f}$ is less than unity, the dipoles of both molecules will be oriented in such a way that the effective dipoles will be less than the corresponding average value of the pure liquids. If the value of $g^{f}$ is greater than unity, the effective dipole in the mixture will be more than the corresponding average value in the pure liquids.

The Bruggemann parameter " $a$ " is the dielectric parameters that can provide information on the interaction between components in the mixture in terms of volume changes (Fig. 7). For present system value of " $a$ " for all temperatures is positive (i.e., $a=1.0474,1.0448$ and 1.0487 at 303.15 , 313.15 and $323.15 \mathrm{~K}$, respectively) and has a smaller deviation from unity. Hence, the interaction in terms of volume changes between the components in the mixture is minimum. ${ }^{33}$ Coefficients of the Redlich-Kister equation for the mathematical representation of deviation in excess functions and the standard deviation $(\sigma)$ calculated for all the excess properties under study for EGMPE + 1-BuOH and EGMPE $+1-\mathrm{BuOH}$ at temperatures $303.15,313.15$ and $323.15 \mathrm{~K}$ are presented in Table 5. 
Table 3. Comparison of the experimental values of refractive index (n) with predicted values by Lorentz-Lorentz (LL), Wiener (W), Gladstone-Dale (GD), Arago-Biot (AB), Eyring-John (EJ), Newton (N) and Heller (H) rules at $T=303.15 \mathrm{~K}$.

\begin{tabular}{lcccccccc}
\hline$\phi_{1}$ & $n_{\text {Exp }}$ & Lorentz-Lorentz & Wiener & Gladstone-Dale & Arago-Biot & Eyring-John (E-J) & Newton & Heller \\
\hline 0.000 & 1.503 & 1.503 & 1.5032 & 1.503 & 1.5032 & 1.503 & 1.503 & 1.5 \\
0.133 & 1.499 & 1.494 & 1.4939 & 1.494 & 1.494 & 1.494 & 1.494 & 1.49 \\
0.256 & 1.494 & 1.484 & 1.4847 & 1.485 & 1.4849 & 1.485 & 1.485 & 1.48 \\
0.371 & 1.488 & 1.475 & 1.4755 & 1.476 & 1.4758 & 1.476 & 1.476 & 1.47 \\
0.479 & 1.481 & 1.466 & 1.4662 & 1.467 & 1.4665 & 1.466 & 1.467 & 1.46 \\
0.579 & 1.473 & 1.456 & 1.4568 & 1.457 & 1.457 & 1.457 & 1.458 & 1.45 \\
0.674 & 1.462 & 1.447 & 1.4478 & 1.448 & 1.448 & 1.448 & 1.449 & 1.45 \\
0.763 & 1.451 & 1.438 & 1.4387 & 1.439 & 1.4389 & 1.439 & 1.44 & 1.44 \\
0.846 & 1.437 & 1.429 & 1.4296 & 1.43 & 1.4298 & 1.43 & 1.43 & 1.43 \\
0.925 & 1.427 & 1.42 & 1.4201 & 1.42 & 1.4202 & 1.42 & 1.421 & 1.42 \\
1.000 & 1.412 & 1.412 & 1.4119 & 1.412 & 1.412 & 1.412 & 1.412 & 1.42 \\
\hline
\end{tabular}

Table 4. RMSD values of different mixing rules for EGMPE + 1-BuOH at $303.15 \mathrm{~K}$.

\begin{tabular}{|c|c|c|c|}
\hline \multicolumn{4}{|c|}{$R M S D$} \\
\hline \multicolumn{2}{|c|}{$303.15 \mathrm{~K}$} & \multicolumn{2}{|c|}{$303.15 \mathrm{~K}$} \\
\hline Mixing rules & Static permittivity $(\varepsilon)$ & Mixing rules & Refractive index $(n)$ \\
\hline Looyenga & 0.176 & LL & 0.0107 \\
\hline Kraszewski & 0.108 & $W$ & 0.0104 \\
\hline Lglesias & 0.147 & $G D$ & 0.0102 \\
\hline Rother & 0.216 & $A B$ & 0.0102 \\
\hline Bottcher & 0.147 & $E J$ & 0.0104 \\
\hline \multirow[t]{2}{*}{ Bruggeman } & 1.058 & $N w$ & 0.0097 \\
\hline & & $H$ & 0.0125 \\
\hline
\end{tabular}

Table 5. Parameters of Redlich-Kister equation for the mathematical representation of deviation in excess functions for EGMPE +1-BuOH.

\begin{tabular}{lccccccl}
\hline Excess properties & Temp (K) & $a_{0}$ & $a_{1}$ & $a_{2}$ & \multicolumn{1}{c}{$a_{3}$} & $a_{4}$ & \multicolumn{1}{c}{$\sigma$} \\
\hline$\varepsilon^{E}$ & 303.15 & -0.4442 & -0.2231 & -0.0060 & 0.04109 & -0.0032 & 0.00008 \\
& 313.15 & -0.3473 & -0.2040 & -0.0376 & 0.06186 & 0.0835 & 0.00009 \\
& 323.15 & -0.2941 & -0.1670 & 0.00254 & 0.1133 & 0.1068 & 0.0026 \\
$\varepsilon_{\infty}^{E}$ & 303.15 & 0.1969 & -0.0444 & -0.0296 & 0.0183 & -0.0221 & 0.0005 \\
& 313.15 & 0.1686 & -0.0808 & -0.0802 & 0.0124 & 0.08078 & 0.0009 \\
& 323.15 & 0.1417 & -0.0827 & -0.0108 & 0.03374 & -0.0100 & 0.0005 \\
\hline
\end{tabular}

\section{Conclusion}

Static permittivity $(\varepsilon)$, density $(\rho)$ and permittivity at optical frequency of binary mixtures of EGMPE and 1-BuOH over complete mixing range at temperatures $(T=303.15,313.15$ and $323.15 \mathrm{~K}$ ) are reported. In the EGMPE rich region, uniform cooperative behavior of the EGMPE-1-BuOH structures was observed whereas in 1-BuOH region, the molecular structures of EGMPE and 1-BuOH are found to differ from their pure liquid state. Negative $\varepsilon^{E}$ values suggest that the polar association forms with lower dipole due to orientation of some of the neighboring dipoles in the opposite direction. H-bonded interaction between the EGMPE and 1-BuOH molecules influences the electronic polarization. H-bonded molecular multimers of pure EGMPE and 1-BuOH, as well as their mixtures favor parallel orientation. Comparison of experimental values of static permittivity and refractive index shows good agreement with theoretically calculated values using mixing rules proposed by Kraszewski and Nw, respectively. 


\section{Acknowledgments}

Experimental facilities developed using financial assistance provided by Department of Sciences and Technology (DST), New Delhi through the DST-FIST (Level- I) project (SR/FST/ PSI-001/2006), University Grant Commission (UGC), New Delhi through major research project (37-159/2009 (SR)) and DRS-SAP program grant [No.F. 530/10/DRS/2010(SAP-I)] have been utilized to carry out this work and it is gratefully acknowledged. Authors are thankful to Prof. P. N. Gajjar, Head, Department of Physics, School of Sciences, Gujarat University, Ahmedabad for his constant encouragement.

\section{References}

${ }^{1}$ A. Chmielewska, M. Zurada, K. Klimasewski and A. Bald, Dielectric properties of methanol mixtures with ethanol, isomers of propanol and butanol, J. Chem. Eng. Data 54, 801 (2009).

${ }^{2}$ R. J. Sengwa, Madhvi, S. Sankhla and S. Sharma. Characterization of heterogeneous interaction behavior in ternary mixtures by a dielectric analysis: Equi-molar $\mathrm{H}$-bonded binary polar mixtures in aqueous, Solutions, J. Sol. Chem. 35, 1037 (2006).

${ }^{3}$ W. Dannhasuser and R H. Cole, Dielectric Properties of Liquid Butyl Alcohols, J. Chem. Phys. 23, 1762 (1955).

${ }^{4}$ T. P. Iglesias and J. Peon Fernandez. A new approach for prediction of the permittivity of mixtures, J. Chem. Thermodyn. 33, 375 (2001).

${ }^{5}$ R. J. Sengwa. Comparative dielectric study of mono, di and trihydric alcohols, Ind. J. Pure Appl. Phys. 41, 295 (2003).

${ }^{6}$ G. A. Ghanadzadeh, H. Ghanadzadeh, K. Bahrpaima and A. Ranjkesh. Dielectric properties of binary mixtures of three butanediols with 1, 4-dioxane and 2-ethyl-1-hexanol at $\mathrm{T}=298.2 \mathrm{~K}$, J. Chem. Therm. 42, 967 (2010).

${ }^{7}$ V. A. Rana, H. A. Chaube and D. H. Gadani, Dielectric permittivity, density, viscosity and refractive index of binary mixtures of anisole with methanol and 1-propanol at different temperatures, J. Mol. Liq. 164, 191 (2011).

${ }^{8}$ V. A. Rana and H. A. Chaube, Relative permittivity, density, viscosity, refractive index and ultrasonic velocity of binary mixture of ethylene glycol mono phenyl ether and 1-hexanol at different temperatures, J. Mol. Liq. 187, 66 (2013).

${ }^{9}$ V. A. Rana, H. A. Chaube, Static permittivity, density, viscosity and refractive index of binary mixtures of anisole with 1-butanol and 1-heptanol at different temperatures, J. Mol. Liq. 173, 71 (2012).

${ }^{10}$ H. A. Chaube, V. A. Rana, P. Hudge and A. C. Kumbharkhane, Dielectric relaxation studies of binary mixture of ethylene glycol mono phenyl ether and methanol by Time Domain Reflectometry, J. Mol. Liq. 193, 29 (2014).

${ }^{11} \mathrm{O}$. Redlich and A. T. Kister, Algebraic representation of thermodynamic properties and the classification of solutions, Ind. Eng. Chem. 140, 345 (1948).

${ }^{12}$ H. Eyring and M. S. Jhon, Significant Liquid Structures (John Wiley \& Sons, New York, 1969).

${ }^{13}$ J. H. Gladstone, T. P. Dale, Researches on the refraction, dispersion, and sensitiveness of liquids, Phil. Trans. R. Soc. London 153, 317 (1863).

${ }^{14} \mathrm{~S}$. Kurtz and A. Ward, The refractivity intercept and the specific refraction equation of newton. I. development of the refractivity intercept and composition with specific refraction equations, $J$. Franklin Inst. 222, 563 (1936).

${ }^{15}$ S. Sharma, P. B. Patel, R. S. Patel and J. Vora, Density and comparative refractive index study on mixing properties of binary liquid mixtures of eucalyptol with hydrocarbons at 303.15, 308.15 and 313.15 K, J. Chem. 4, 343 (2007).

${ }^{16} \mathrm{~W}$. Heller, The determination of refractive indices of colloidal particles by means of a new mixture rule or from measurements of light scattering, Phys. Rev. 68, 5 (1945).

${ }^{17}$ H. Looyenga, Dielectric constants of heterogeneous mixtures. Physica 31, 401 (1965).

${ }^{18}$ J. F. Peón-Fernández and T. P. Iglesias, On the permittivity of mixtures: A low-fluctuation approach, J. Electrostat. 32, 113 (1994).

${ }^{19}$ A. Kraszewski, S. Kulinski and M. Matuszewski, Dielectric properties and a model of biphase water suspension at $9.4 \mathrm{GHz}$, J. Appl. Phys. 47, 1275 (1976).

${ }^{20} \mathrm{C}$. Böttcher and P. Bordewijk, Theory of Electrical Polarization, 2nd edn. (Elsevier, Amsterdam, 1978).

${ }^{21}$ D. Bruggeman, Dielectric constant and conductivity of mixtures of isotropic materials, Ann. Phys. 24, 636 (1935).

${ }^{22} \mathrm{~K}$. Lichtenecker and K. Rother, Die Herleitung des logarithmischen Mischungsgesetzes aus allgemeinen Prinzipien der stationären Strömung, PHYS. Z 32, 255 (1931).

${ }^{23} \mathrm{~V}$. A. Rana and D. H. Gadani, Dielectric properties of mixtures of bromobenzene and 1-heptanol at RT, IJAECE 2, 252 (2012).

${ }^{24}$ C. Campbell, J. Crossely and L. Glasser, Dielectric relaxation of some alcohols in solution, Adv. Mole. Relax. Process. 9, 63 (1976).

${ }^{25}$ M. I. Aralaguppi, C. V. Jadar and T. M. Aminabhavi, Density, viscosity, refractive index, and speed of sound in binary mixtures of acrylonitrile with methanol, ethanol, propan-1-ol, butan-1-ol, pentan-1-ol, hexan-1-ol, heptan-1-ol, and butan-2-ol, J. Chem. Eng. Data 44, 216 (1999).

${ }^{26}$ W. L. Weng, Viscosities and densities for binary mixtures of anisole with 1-butanol, 1-pentanol, 1-hexanol, 1-heptanol, and 1octanol, J. Chem. Eng. Data 44, 63 (1999).

${ }^{27}$ TRC Thermodynamic Tables, Non-Hydrocarbons; Thermodynamics Research Center (The Texas A \& M University System, College Station, TX, 1994).

${ }^{28}$ T. S. Vijayalakshmi and P. R. Naidu, Excess volumes of binary mixtures of 1,2,4-trichlorobenzene with 1-alkanols, J. Chem. Eng. Data 37, 368 (1992)

${ }^{29}$ A. Chaudhari, S. Ahire and M. Lokhande, Dielectric study of pyridine-alcohol binary liquids at $25^{\circ} \mathrm{C}$, Proc. Natl. Acad. Sci. India A 1, 75 (2001).

${ }^{30}$ R. J. Sengwa, V. Khatri and S. Sankhla, Dielectric behavior and hydrogen bond molecular interaction study of formamide-dipolar solvents binary mixtures, J. Mol. Liq. 144, 89 (2009).

${ }^{31}$ R. J. Sengwa, Madhvi and S. Sankhla, H-bonded molecular interaction study on binary mixtures of mono alkyl ethers of ethylene glycol with different polar solvents by concentration dependent dielectric analysis, Phys. Chem. Liq. 44, 637 (2006).

${ }^{32}$ D. Balamurugan, S. Kumar and S. Krishnan, Dielectric relaxation studies of higher order alcohol complexes with amines using time domain reflectometry, J. Mol. Liq. 122, 11 (2005).

${ }^{33}$ T. Thenappan and A. Prabakar Devaraj, Dielectric studies on binary polar mixtures of propanoic acid with esters, J. Mol. Liq. 123, 72 (2006). 